\title{
Estudo da Transmissão da Dengue entre os Indivíduos em Interação com a População de Mosquitos Aedes Aegypti
}

C.P. FERREIRA ${ }^{1}$, H.M. YANG ${ }^{2}$, Departamento de Matemática Aplicada, Instituto de Matemática, Estatística e Computação Científica, UNICAMP, Cx.P. 6065, 13081-970 Campinas, SP, Brasil.

Resumo. Estuda-se a dinâmica da transmissão da dengue na população humana acoplada à dinâmica do vetor mosquito. Considera-se a circulação de um único sorotipo na comunidade, sendo que as populações humana e de mosquitos são descritas por um modelo determinístico compartimental. Supondo que o encontro entre os infectantes e os suscetíveis acontece de maneira aleatória, estuda-se o efeito da ação dos mecanismos de controle (aplicados na população de mosquitos) sobre a doença para diferentes valores das forças de infecção.

\section{Introdução}

A dengue é causada por um arbovírus, tendo o mosquito Aedes aegypti como vetor. Esta infecção nos mosquitos não encurta a vida média e nem cria imunidade. Entretanto, na população humana, ela induz a uma imunidade perene e, no caso da circulação de um único sorotipo na comunidade, os indivíduos sintomáticos desta doença não são levados à morte. A dinâmica de transmissão da dengue envolve, portanto, um vírus e duas populações, a de mosquitos e a humana, as quais serão descritas através de um modelo determinístico compartimental. O acoplamento entre as populações é feito através das forças de infecção e considera-se mecanismos de controle agindo apenas sobre a população de mosquitos. Os resultados analíticos obtidos em [6] consideram os parâmetros constantes no tempo e mostram que a erradicação da dengue na população humana pode ser obtida através de mecanismos de controle sobre o vetor. Aqui, considera-se a dependência temporal nos tempos de desenvolvimento e sobrevida das fases do mosquitos com a temperatura e umidade [3], e avalia-se a eficiência da aplicação periódica de controle feita na população de mosquitos sobre a doença. Os resultados númericos são obtidos pelo método de Runge-Kutta de $4^{a}$ ordem.

\footnotetext{
${ }^{1}$ pio@ime.unicamp.br (apoio financeiro FAPESP)

${ }^{2}$ hyunyang@ime.unicamp.br (apoio financeiro FAPESP e CNPq)
} 


\section{Modelo}

Com relação à população de mosquitos, considera-se as 4 fases do ciclo de vida do vetor, a saber: ovo $(E)$, larva $(L)$, pupa $(P)$ e adulto $(W)$, sendo que a população adulta é dividida em mosquitos suscetíveis, $W_{1}$, infectados porém não infectantes, $W_{2}$, e infectantes, $W_{3}$, de modo que a dinâmica desta população é dada por:

$$
\begin{aligned}
\frac{d}{d t} E(t) & =\phi(t) F(W)\left[1-\frac{E(t)}{(1-f) C}\right]-\left[\sigma_{e}(t)+\mu_{e}(t)+m_{e}(t)\right] E(t) \\
\frac{d}{d t} L(t) & =\sigma_{e}(t) L(t)-\left[\sigma_{l}(t)+\mu_{l}(t)+\mu_{l}^{\prime}(t)+m_{l}(t)\right] L(t) \\
\frac{d}{d t} P(t) & =\sigma_{l}(t) L(t)-\left[\sigma_{p}(t)+\mu_{p}(t)+\mu_{p}^{\prime}(t)+m_{p}(t)\right] P(t) \\
\frac{d}{d t} W_{1}(t) & =\sigma_{p}(t) P(t)-\left[\eta_{w}(I)+\mu_{w}(t)+\mu_{w}^{\prime}(t)\right] W_{1}(t) \\
\frac{d}{d t} W_{2}(t) & =\eta_{w}(I) W_{1}(t)-\left[\gamma_{w}+\mu_{w}(t)+\mu_{w}^{\prime}(t)+\mu_{2}(t)\right] W_{2}(t) \\
\frac{d}{d t} W_{3}(t) & =\gamma_{w} W_{2}(t)-\left[\mu_{w}(t)+\mu_{w}^{\prime}(t)+\mu_{3}(t)\right] W_{3}(t) .
\end{aligned}
$$

Os parâmetos $\sigma_{e}, \sigma_{l}$ e $\sigma_{p}$ são as taxas de transição entre os compartimentos (inverso do tempo de desenvolvimento de cada fase); $\mu_{e}, \mu_{l}, \mu_{p}, \mu_{w}, \mu_{2}$ e $\mu_{3}$ são as taxas de mortalidades de cada fase; $\mu_{l}^{\prime}, \mu_{p}^{\prime}, \mu_{w}^{\prime}, m_{e}, m_{l}$ e $m_{p}$ são as taxas de mortalidade adicionais devido ao controle; $C$ é a capacidade do meio e $f$ é a fração de criadouros retirados durante o controle mecânico. Os resultados apresentados são para a dinâmica em que a capacidade de oviposição das fêmeas depende linearmente da quantidade de população de mosquitos, isto é, $F(W)=W$, e $\phi$ é a taxa de oviposição. Com relação à transmissão da doença, $\gamma_{w}^{-1}$ é o período médio de incubação extrínseca do vírus da dengue no mosquito e $\eta_{w}(I)$ como força de infecção.

A população humana total $N$ é considerada constante. Assim, os indivíduos são subdivididos em quatro compartimentos não interceptantes designados suscetíveis $(s)$, expostos $(h)$, infectados $(i)$ e recuperados $(r)$, de modo que a dinâmica desta população descrita em termos de frações de indivíduos é dada por:

$$
\begin{aligned}
\frac{d}{d t} s(t) & =\mu_{h}-\left[\eta_{h}\left(W_{3}\right)+\mu_{h}\right] s(t) \\
\frac{d}{d t} h(t) & =\eta_{h}\left(W_{3}\right) s(t)-\left[\gamma_{h}+\mu_{h}\right] h(t) \\
\frac{d}{d t} i(t) & =\gamma_{h} h(t)-\left[\sigma_{h}+\mu_{h}\right] i(t) \\
\frac{d}{d t} r(t) & =\sigma_{h} i(t)-\mu_{h} r(t) .
\end{aligned}
$$

Os parâmetros $\mu_{h}, \gamma_{h}^{-1}, \sigma_{h}^{-1}$ e $\eta_{h}\left(W_{3}\right)$ são, respectivamente, a taxa de mortalidade, o período médio de incubação do vírus no homem, o período infeccioso médio do homem e a força de infecção. 
Supoẽ-se que o encontro entre os infectantes e suscetíveis acontece de maneira aleatória, de modo que as relações entre as forças de infecção e as taxas de contato são dadas pelo encontro das massas:

$$
\eta_{w}(I)=\beta_{w} I, \quad \eta_{h}\left(W_{3}\right)=\beta_{h} W_{3},
$$

onde $I=i N$ é o número de indivíduos infectantes e $\beta_{w}$ e $\beta_{h}$ são as taxas de contato per-capita entre indivíduos infectantes (mosquito e homem) e suscetíveis (homem e mosquito). A decrição detalhada do modelo e dos parâmetros encontra-se em [6].

Se os parâmetos do modelo não dependem do tempo, isto é, $\sigma_{e}(t)=\sigma_{e}, \sigma_{l}(t)=$ $\sigma_{l}, \mu_{e}(t)=\mu_{e}$, etc., após um transiente, as populações atingem seus valores de equilíbrio e pode-se identificar três soluções de estado estacionário:

(1) se $\phi<\phi_{t h}=\left(\frac{\sigma_{e}}{\rho_{e}} \frac{\sigma_{l}}{\rho_{l}} \frac{\sigma_{p}}{\rho_{p}} \frac{1}{\rho_{w}}\right)$ tem-se a população humana livre de mosquitos (solução nula, $W=0$ );

(2) se $\phi>\phi_{t h}$ e $R^{\prime}<1$ tem-se população humana infestada por mosquitos sem a transmissão da dengue (solução trivial, $h=0$ );

(3) se $R^{\prime}>1$ tem-se população humana infestada por mosquitos com a transmissão da dengue (solução não-trivial, ou equilíbrio endêmico).

O parâmetro $\phi_{t h}$ é a taxa de oviposição limiar e $R^{\prime}$ representa a razão de reprodutibilidade da dengue. A determinação dos pontos de equilíbrio e análise de estabilidade para o modelo autônomo encontra-se em [6].

\section{Resultados Numéricos}

Inicialmente, analisa-se a evolução da dinâmica da população de mosquitos e humana, sem considerar os mecanismos de controle, como eliminação dos criadouros e aplicação de larvicidas e adulticias, de modo que os resultados mostrados correspondem à situação $f=m_{e}(t)=m_{l}(t)=m_{p}(t)=0$ e $\mu_{l}^{\prime}(t)=\mu_{p}^{\prime}(t)=\mu_{w}^{\prime}(t)=0$.

\subsection{Sem controle}

Considera-se a situação em que uma comunidade livre da doença, isto é, $W_{2}^{(0)}=$ $W_{3}^{(0)}=h^{(0)}=r^{(0)}=0$, entra em contato com a mesma, via a introdução de indivíduos infectados na comunidade em $t=0$. A tabela 1 contém as demais condições iniciais para a população de mosquitos e humana, e os valores dos parâmetros utilizados nas simulações.

A dependência das soluções (2) e (3) do modelo autônomo com os parâmetros $\beta_{w}$ e $\beta_{h}$, que caracterizam as forças de infecção, está mostrada na Figura 1. Para $\beta_{w}=0,005$ e $\beta_{h} \leq 0,002$ em dias ${ }^{-1}$ a solução de equilíbrio corresponde à situção (2), isto é, população humana infestada por mosquitos sem a transmissão da dengue; enquanto para $\beta_{w}=0,005$ e $\beta_{h}>0,002 \mathrm{em} \mathrm{dias}^{-1}$ tem-se a solução (3), isto 
Tabela 1: Condições iniciais e valores dos parâmetros utilizados nas simulações. Os demais parâmetros são: $C=10, \phi=1 \operatorname{dias}^{-1}$ e $\mu_{h}^{-1}=24000$ dias.

\begin{tabular}{|c|c|c|c|c|c|}
\hline$E^{(0)}$ & $L^{(0)}$ & $P^{(0)}$ & $W_{1}^{(0)}$ & $s^{(0)}$ & $i^{(0)}$ \\
\hline 0,2 & 0,1 & 0,2 & 0,9 & 0,999 & 0,001 \\
\hline$\mu_{e}^{-1}($ dias $)$ & $\mu_{l}^{-1}($ dias $)$ & $\mu_{p}^{-1}($ dias $)$ & $\mu_{w}^{-1}($ dias $)$ & $\mu_{2}^{-1}($ dias $)$ & $\mu_{3}^{-1}($ dias $)$ \\
\hline 100 & 1,5 & 4,6 & 17,5 & 70 & 150 \\
\hline$\gamma_{h}^{-1}($ dias $)$ & $\gamma_{w}^{-1}($ dias $)$ & $\sigma_{h}^{-1}($ dias $)$ & $\sigma_{e}^{-1}($ dias $)$ & $\sigma_{l}^{-1}($ dias $)$ & $\sigma_{p}^{-1}($ dias $)$ \\
\hline 10 & 9,5 & 6 & 4,5 & 11,7 & 4,6 \\
\hline
\end{tabular}

é, população humana infestada por mosquitos com a transmissão da dengue. A existência de pontos críticos e a sua dependência com as forças de infecção são muito importantes do ponto de vista de controle e classificação da infecção.
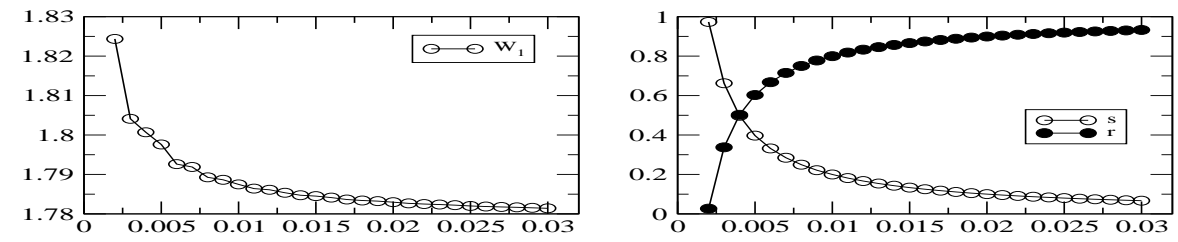

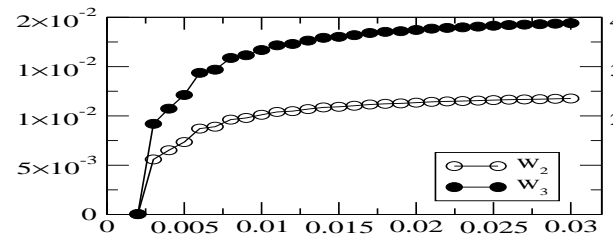

$\beta_{h}$

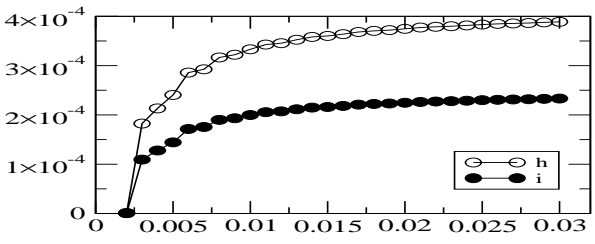

$\beta_{h}$

Figura 1: Soluções de equilíbrio para a população de mosquitos e humana dado $\beta_{w}=$ 0,005 dias $^{-1}$ e diferentes valores de $\beta_{h}$.

As variações abióticas são introduzidas no modelo considerando o ano calendário dividido em apenas dois períodos: temperatura e umidade baixas (período desfavorável) e altas (período favorável). Supõe-se o período desfavorável compreendendo a maior parte do ano, com os parâmetros constantes dados na tabela 1; e o período favorável compreendendo um intervalo de tempo da ordem de 75 dias que corresponde ao período de chuvas entre janeiro/abril (vide [1]). Durante este período as taxas de desenvolvimento e sobrevida podem assumir um dos conjuntos distintos de valores dados na tabela 2 , associados a duas temperaturas.

Nas simulações associa-se uma probabilidade de sorteio $P_{t}$ e $1-P_{t}$ a cada um 
Tabela 2: Média do tempo de desenvolvimento e sobrevida de cada estágio para dois valores de temperatura (em dias).

\begin{tabular}{|c|c|c|c|c|c|}
\hline$T\left({ }^{\circ} \mathrm{C}\right)$ & $\sigma_{e}^{-1}($ dias $)$ & $\sigma_{l}^{-1}($ dias $)$ & $\sigma_{p}^{-1}($ dias $)$ & $\mu_{l}^{-1}($ dias $)$ & $\mu_{m}^{-1}($ dias $)$ \\
\hline 25 & 3,3 & 8 & 3,1 & 2,3 & 26 \\
\hline 27 & 2 & 4,4 & 1,6 & 3 & 35 \\
\hline
\end{tabular}

desses conjuntos, em que a cada 360 passos de tempo ( 1 ano de calendário) um número aleatório $z \in[0,1]$ é sorteado e comparado com $P_{t}$. Se $z \leq P_{t}$ escolhe-se o conjunto de parâmetros relativo a temperatura de $25^{\circ} \mathrm{C}$, se não, a de $27^{\circ} \mathrm{C}$. Utilizouse $P_{t}=0,75$ para mimetizar uma brusca variação ocorrendo esporadicamente $(25 \%$ dos casos). A evolução temporal das diferentes populações é apresentada nas Figuras 2 (população de mosquitos) e 3 (população humana) para o conjunto de parâmetros dados nas tabelas 1 e 2 , com $\beta_{w}=0,013$ e $\beta_{h}=0,01 \mathrm{em} \mathrm{dias}^{-1}$.
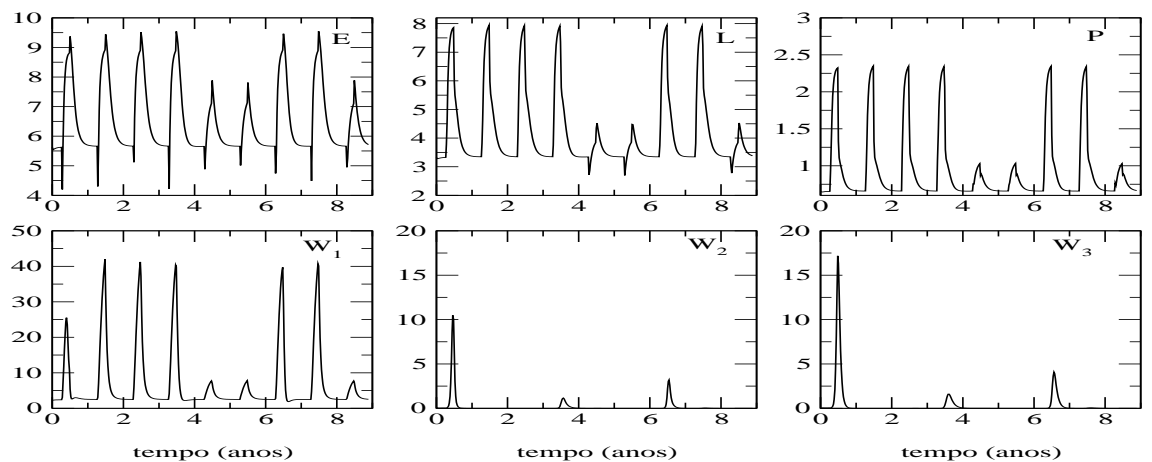

Figura 2: Evolução temporal para a população de mosquitos $\operatorname{com} \beta_{w}=0,013$ e $\beta_{h}=0,01$ em $\operatorname{dias}^{-1}$.

Os picos maiores observados para o número de ovos $(E)$, larvas $(L)$ e pupas $(P)$ correspondem a temperatura de $27^{\circ} \mathrm{C}$ enquanto as menores, à temperatura de $25^{\circ} \mathrm{C}$. Os mosquitos suscetíveis são infectados quando picam indivíduos infectantes, de forma que os picos que aparecem em $W_{2}$ resultam do encontro dessas duas populações. Como conseqüência do período de incubação do vírus no mosquito, os picos que aparecem em $W_{3}$ estão deslocados em relação aos seus correspondentes em $W_{2}$ (retardo).

Em relação à dinâmica da população humana, pode-se observar, na Figura 3, que os picos de infecção aparecem sempre que ocorre uma mudança no comportamento (diminuição) da população de suscetíveis. Os indivíduos suscetíveis são infectados pelas picadas de mosquitos infectantes, de forma que os picos que aparecem em 

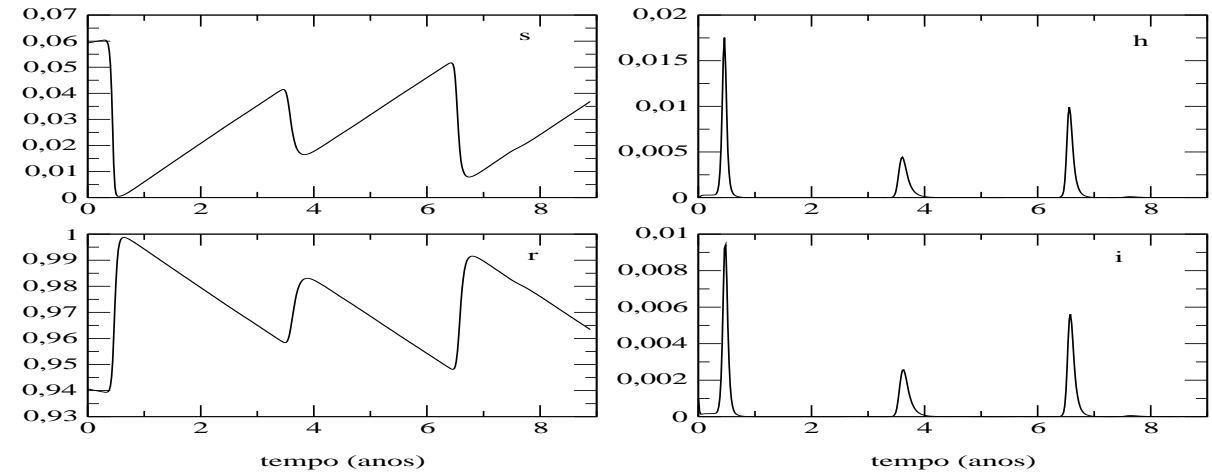

Figura 3: Evolução temporal para a população humana com $\beta_{w}=0,013$ e $\beta_{h}=0,01$ em $\operatorname{dias}^{-1}$.

$h$ são resultados do encontro entre essas duas populações. Devido ao período de incubação do vírus no homem, os picos que aparecem em $i$ estão deslocados em relação aos seus correspondentes em $h$. Estes deslocamentos não são observados nas figuras por causa da escala do tempo (em anos).

Quanto maior o valor de $\sigma_{h}^{-1}$, tempo durante o qual o indivíduo infectante transmite o vírus, maior é o número de indivíduos infectados em um mesmo período de tempo como pode ser visto na Figura 4. A possibilidade de uma nova infecção aumenta também quando a população cresce, pois neste caso ocorrerá maior número de contatos entre indivíduos/mosquitos infectantes e mosquitos/indivíduos suscetíveis.
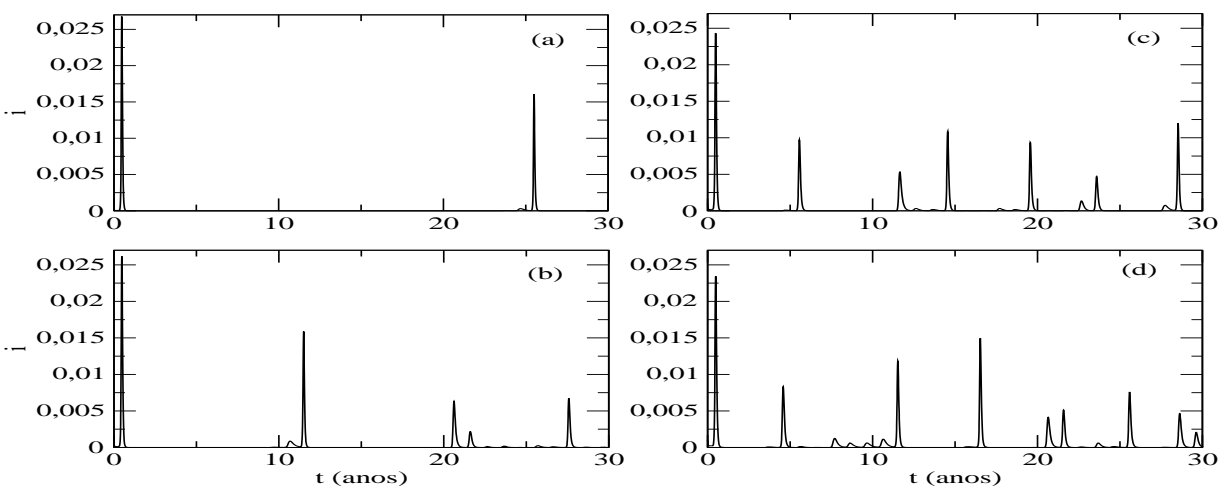

Figura 4: Variação da densidade de indivíduos infectados com $\sigma_{h}^{-1}$. Tem-se em (a), (b), (c) e (d), respectivamente, $\sigma_{h}^{-1}=2,5,8$ e 10 em dias. 

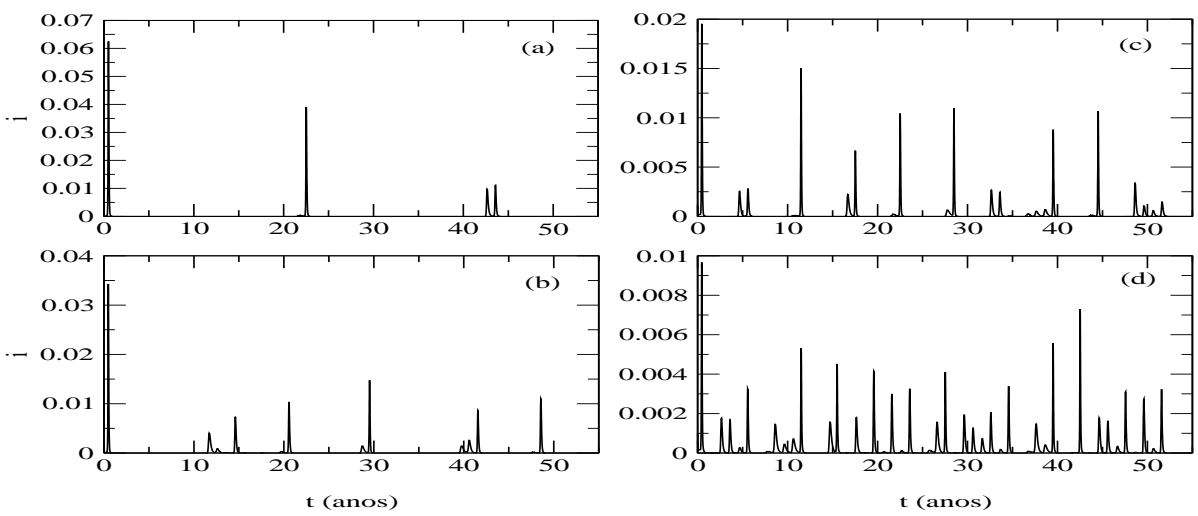

Figura 5: Variação com o produto $\beta_{w} \beta_{h}$ que mede a força da infecção. Tem-se em (a), (b), (c) e (d), respectivamente, $\beta_{w} \beta_{h}=2,5 \times 10^{-5} ; 0,5 \times 10^{-4} ; 1 \times 10^{-3}$ e $2 \times 10^{-3} \mathrm{em}$ $\operatorname{dias}^{-2}$

O mesmo resultado é obtido quando se varia o produto $\beta_{w} \beta_{h}$ que mede a força de infecção. À medida que a epidemia torna-se mais grave, menor é o espaçamento entre os dois picos suscessivos, como pode ser visto na Figura 5. Com relação aos tempos de incubação do vírus no homem e no mosquito, o comportamento é oposto ao descrito anteriormente: o aumento de $\gamma_{w}$ e/ou $\gamma_{h}$ acarreta a diminuição do número de suscetíveis de forma que dois picos sucessivos de infecção estão mais afastados para acumular os suscetíveis para valores limiares.

Portanto, existem picos de epidemias anuais devido à variação dos parâmetros (a escala em anos do gráfico dificulta a visualização) e picos maiores em intervalos superiores a um ano que dependem, por exemplo, dos valores de $\beta_{w}$ e $\beta_{h}$. Estes picos epidêmicos mais acentuados devem-se ao acúmulo de suscetíveis.

\subsection{Com controle}

Os mecanismos de controle são introduzidos como taxas de mortalidade adicionais em cada uma das fases em que atuam. Para medir a eficiência de cada um deles, compara-se a área das curvas obtidas para $W_{1}, W_{2}, W_{3}$ e $i$, quando a simulação é feita na ausência $\left(A_{0}\right)$ e na presença $\left(A_{1}\right)$ de algum mecanismo de controle (vide [1]). Os resultados mostrados indicam a porcentagem de mosquitos mortos, $J_{w}=100 \times$ $\left(1-A_{1}^{w} / A_{0}^{w}\right)$, ou humanos protegidos, $J_{i}=100 \times\left(1-A_{1}^{i} / A_{0}^{i}\right)$, devido à aplicação periódica do controle durante o intervalo de tempo $\Delta t$ em anos. Ressalta-se que o estudo da ação dos diferentes mecanismos de controle é feito individualmente, por exemplo, o efeito de adulticida é medido pelo parâmetro $\mu_{w}^{\prime}$ fazendo-se os demais parâmetros, $f, m_{e}, m_{l}, m_{p}, \mu_{e}^{\prime}$ e $\mu_{l}^{\prime}$ iguais a zero.

Nas Figuras 6 e 7 pode-se ver a eficiência obtida devido à aplicação de adulticida 

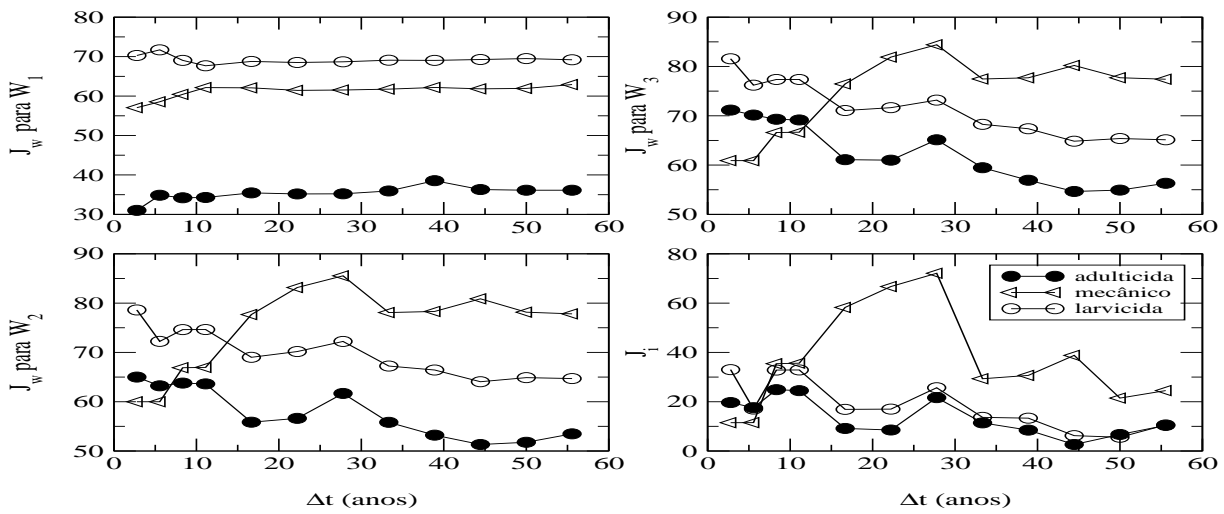

Figura 6: Comparação entre a aplicação de adulticida $(\bullet), \mu_{w}^{\prime}=1$ dias $^{-1}$, larvicida (o), $\mu_{l}^{\prime}=5 \operatorname{dias}^{-1}$ e $\mu_{p}^{\prime}=2 \operatorname{dias}^{-1}$, e controle mecânico $(\triangleleft), m_{e}=m_{l}=m_{p}=0,1$ em dias ${ }^{-1}$ e $f=0,4$ para $t_{i}=110$ dias, $\beta_{w}=0,013$ e $\beta_{h}=0,005 \mathrm{em} \mathrm{dias}^{-1}$.

(durante 10 dias), larvicida (durante 50 dias) e o controle mecânico para dois valores das forças de infecção. Pode-se observar que o padrão das curvas $J_{w}$ e $J_{i}$ mostrado nas figuras é semelhante, o que leva a concluir que variações nas forças de infecção não traz muitas mudanças qualitativas.

Para uma mesma taxa de mortalidade adicional (inseticida ou larvicida) e remoção mecânica dos criadouros, a eficácia é diferente em $W_{1}, W_{2}$ e $W_{3}$, sendo maior para $W_{2}$ e $W_{3}$. Este comportamento deve-se a quantidade das populações (vide Figura 2 ), pois $W_{1}$ é muito maior que $W_{2}$ e $W_{3}$, e, portanto, menos sensitivo. A mesma explicação se aplica parcialmente à eficácia $J_{i}$, pois $i$ é muito pequeno.

Ainda com relação a população de mosquitos, pode-se observar que a eficácia $J_{w}$ para $W_{3}$ e $W_{2}$ oscila e diminui com o tempo de aplicação de inseticida e larvicida enquanto que para $W_{1}$ mantem-se praticamente constante. No caso do controle mecânico, a eficácia $J_{w}$ para $W_{1}, W_{2}$ e $W_{3}$ sempre aumenta, devido ao fato de não haver reposição de criadouros, que diminui a capacidade de manter a infestação.

A eficácia $J_{i}$ para indivíduos infectantes oscila e diminui com o tempo, sendo que, para forca̧s de infecção elevadas é praticamente nula. Uma possível explicação para este comportamento é que a introdução de mecanismos de controle protege a população humana (suscetíveis), de maneira que os picos de infecção ocorrem mais tarde, mais espaçados e, as vezes, com mais intensidade. Este deslocamento dos picos de infecção da simulação com controle em relação a simulação sem controle gera a dependência observada com o tempo de aplicação do controle, e tem como consequência a diminuição da proteção (número de mosquitos mortos e indivíduos protegidos) para tempos grandes, pois a intensidade da aplicação é sempre a mesma.

Ressalta-se que, se os parâmetros que definem o modelo não dependem do tempo (sistema autônomo), então não há dependênciade $J$ com o tempo de aplicação $\Delta t$. 

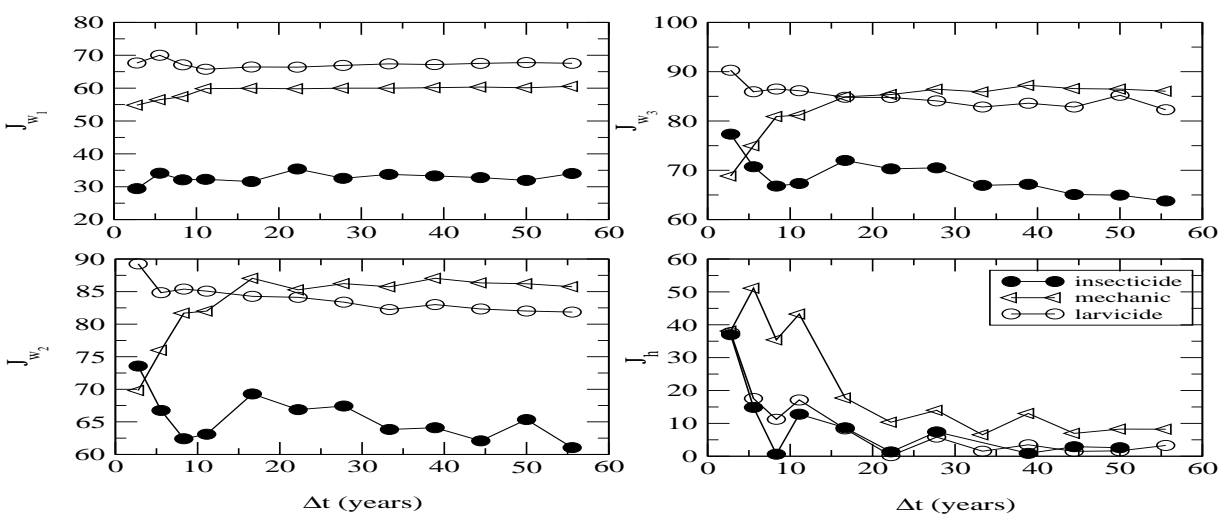

Figura 7: Comparação entre a aplicação de adulticida $(\bullet), \mu_{w}^{\prime}=1 \operatorname{dias}^{-1}$, larvicida (o), $\mu_{l}^{\prime}=5 \operatorname{dias}^{-1}$ e $\mu_{p}^{\prime}=2 \operatorname{dias}^{-1}$, e controle mecânico $(\triangleleft), m_{e}=m_{l}=m_{p}=0,1 \mathrm{em} \mathrm{dias}^{-1}$ e $f=0,4$ para $t_{i}=110 \operatorname{dias}^{-1}, \beta_{w}=0,013$ e $\beta_{h}=0,02 \mathrm{em} \mathrm{dias}^{-1}$.

\section{Conclusão}

No modelo autômato [6] a erradicação da dengue na população humana é sempre possível, basta que se diminua a razão de reprodutibilidade da transmissão da dengue, $R^{\prime}$, para valores abaixo da unidade por alguma forma de controle aplicada na população de mosquitos. O parâmetro $R^{\prime}$ depende explicitamente das taxas de contato, daí a importância da determinação dos valores críticos para $\beta_{h}$ e $\beta_{w}$, pois quanto maior forem estes valores críticos, maior será a dificuldade para a epidemia se perpetuar. Para, também, entender o porquê da incidência da doença ser maior em áreas com alta densidade populacional, basta supor que $\eta_{w}(I)=\varepsilon \beta_{w} I$ e $\eta_{h}(I)=\varepsilon \beta_{h} W_{3}$, onde o parâmetro $\varepsilon$ mede a forma como os indivíduos estão distribuídos geograficamente, facilitando ou não a transmissão da dengue. Para valores fixos de $\beta_{w}$ e $\beta_{h}$, variações no parâmetro $\varepsilon$ deslocam os picos de infecção suscessivos, deixando-os mais próximos ou mais afastados.

As variações abióticas foram consideradas no modelo através da introdução da dependência temporal nos períodos de desenvolvimento e sobrevida das diferentes fases do ciclo de vida do mosquito. Os resultados mostram que o controle com a mesma intensidade aplicado periodicamente (e intermitentemente) pode não conseguir atingir nenhum dos objetivos que são: a erradicação da doença ou a diminuição dos novos casos de infecção. Isto porque o deslocamento dos picos de infecção ocasionado pelo controle gera, a longo prazo, uma diminuição na eficiência deste mecanismo, devido à existência de picos de incidência que surgem bruscamente no tempo e na intensidade. Assim, o controle deve ser feito de maneira periódica, porém com intensidades diferentes para levar em consideração esses picos de infecção intensos ocasionados pelo acúmulo de indivíduos suscetíveis. 
Em regime estacionário, no caso do sistema autônomo, pode-se determinar o valor de $R^{\prime}$ a partir dos parâmetros que definem o modelo, e a introdução de mecanismos de controle visa diminuir este parâmetro para valores menores que a unidade, de modo que a erradicação da doença seja possível. Fundamentados em modelos homogêneos, mecanismos de controle têm sido propostos, seja para dengue, seja para outras infecções. Entretanto, os controles têm sido fugazes para obter seus objetivos. Este trabalho mostrou que, quando se considera a dependência temporal devido às variações abióticas (modelo não-autônomo), os resultados do controle dependem do tempo de aplicação. Além desta sazonalidade de aplicação de controle, pode-se ter a manutenção da doença devido ao fato de se ter durante o período favorável uma capacidade propagação de doença aumentada (como se tivesse $R^{\prime}>1$ ), mesmo que em outros períodos se tenha esta propagação dificultada (como se tivesse $R^{\prime}<1$ ). Por isso, quando se leva em consideração fatores não-homogêneos (como variações sazonias), mostra-se que o controle periódico deve ser acompanhado de um estudo da dinâmica da doença para que possa identificar períodos de baixa endemicidade e se possa fazer um controle mais intenso a fim de evitar epidemias devido ao acúmulo de suscetíveis.

\begin{abstract}
The dynamics of dengue transmission is assessed considering the population dynamics applied to humans and mosquitos. Compartimental model is developed taking into account the random encounter between susceptible and infectious individuals in order to analize the erdication of dengue disease by the controlling mechanisms applied to the mosquito population.
\end{abstract}

\title{
Referências
}

[1] C.P. Ferreira e H.M. Yang, Estudo dinâmico da população de mosquitos Aedes aegupti, em "Seleta do XXV CNMAC" (E.X.L. de Andrade et al., eds.), TEMA Tend. Mat. Apl. Comput., 4, No. 2 (2003), no prelo.

[2] R. Veronesi, "Doenças Infecciosas e Parasitárias", Oitava Edição, Guanabara Koogan, Rio de Janeiro, 1991.

[3] H.M. Yang e M.U. Ferreira, Assessing the effects of global warming and local socio-economic conditions on the malaria transmission by a mathematical model, Rev. Saúde Pública 34, No. 3 (2000), 214-222.

[4] H.M. Yang, "Epidemiologia Matemática-Estudos dos efeitos da Vacinação em Doenças de Transmissão Direta", EDUNICAMP e FAPESP, Campinas e São Paulo, 2001.

[5] H.M. Yang, C.P.Ferreira e S. Ternes, Dinâmica populacional do vetor transmissor da dengue, em "Seleta do XXV CNMAC" (E.X.L. de Andrade et al., eds.), TEMA Tend. Mat. Apl. Comput., 4, No. 2 (2003), no prelo.

[6] H.M. Yang, Epidemiologia da transmissão da dengue, em "Seleta do XXV CNMAC" (E.X.L. de Andrade et al., eds.), TEMA Tend. Mat. Apl. Comput., 4, No. 2 (2003), no prelo. 